\title{
COMPORTAMIENTO AGRESIVO Y PROSOCIAL DE ESCOLARES RESIDENTES EN ENTORNOS CON ALTOS NIVELES DE POBREZA
}

\author{
José William Martínez ${ }^{1, a}$, José Rafael Tovar Cuevas²,b, Andrés Felipe Ochoa Muñoz ${ }^{3, c}$
}

\begin{abstract}
RESUMEN
Objetivos. Evaluar los niveles de agresividad directa e indirecta y prosocialidad en un grupo de escolares de colegios públicos ubicados en trece municipios de un departamento intermedio de Colombia. Materiales y métodos. Un total de 2785 niños y niñas con edades entre los tres y once años fueron evaluados con un instrumento validado para población colombiana, que mide agresividad y prosocialidad a través de la observación directa del maestro de escuela. Resultados. Los niños presentaron predominantemente comportamientos asociados con la agresividad directa $(22,4 \%)$ y las niñas a la agresividad indirecta $(8,0 \%)$. Los comportamientos prosociales fueron mas comunes en las niñas $(67,2 \%)$ y en las edades de 10 y 11 años de edad $(67,4 \%)$. Conclusiones. El grado de presencia de comportamientos agresivos estaría relacionada con el contexto sociocultural en el que viven los niños y esta problemática puede ser detectada e intervenida por los maestros en la escuela.
\end{abstract}

Palabras claves: Agresividad directa; Agresividad indirecta; Prosocialidad; Niños (fuente: DeCS BIREME).

\section{AGGRESSIVE AND PROSOCIAL BEHAVIORS AMONG SCHOOLCHILDREN LIVING IN ENVIRONMENTS WITH HIGH LEVELS OF POVERTY}

\begin{abstract}
Objetives. To assess the levels of direct and indirect aggression and prosocial behaviors among schoolchildren at public schools located in 13 municipalities of an intermediate region of Colombia. Materials and Methods. A total of 2,785 children aged 3-11 years were assessed using an instrument validated for the Colombian population that measures aggression and prosocial behavior via direct observations of schoolteachers. Results. Boys predominantly exhibited behaviors associated with direct aggression (22.4\%), whereas girls exhibited behaviors of indirect aggression (8.0\%). Prosocial behaviors were more common in women (67.2\%) and in 10-11-year-old children (67.4\%). Conclusions. The degree of aggressive behavior may be related to the sociocultural milieu in which children live, and can be detected and subjected to intervention by schoolteachers.
\end{abstract}

Key words: Direct aggressiveness; Indirect aggressiveness; Prosociality; Child (source: MeSH NLM).

\section{INTRODUCCIÓN}

Un importante predictor de conductas violentas en el joven y en el adulto es el comportamiento agresivo infantil. Identificar el origen del comportamiento agresivo infantil y desarrollar actividades que promuevan las conductas que se oponen al individualismo, la competitividad y la insensibilidad hacia los problemas ajenos, facilitan la presencia de formas asertivas de comunicación y de autocontrol, lo que, a largo y mediano plazo, no solo disminuye los costos mundiales debidos a los actos violentos ${ }^{(1-3)}$, sino que también los índices de delincuencia, robo, mentira, vandalismo, promiscuidad sexual, y consumo de alcohol en adultos ${ }^{(4-7)}$. Algunos investigadores ${ }^{(8,9)}$ han incluido los comportamientos agresivos entre las comorbilidades asociadas a problemas como el déficit de atención, la depresión, ansiedad y la oposición desafiante, por lo que la agresividad infantil es ahora un asunto de interés para médicos y salubristas. Es así como, el Centro de Control de Enfermedades de Atlanta, ha propuesto intervenciones cuyo blanco de acción son los niños en proceso en formación escolar, con el objetivo de reducir las lesiones, la trayectoria de delitos que empieza en la infancia, el cambio frecuente de

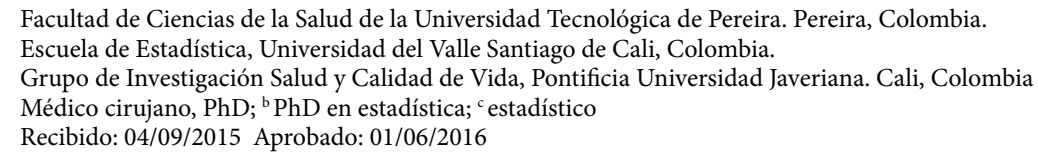

Citar como: William Martínez J, Tovar Cuevas JR, Ochoa Muñoz AF. Comportamiento agresivo y prosocial de escolares residentes en entornos con altos niveles de pobreza. Rev Peru Med Exp Salud Publica. 2016;33(3):455-61. doi: 10.17843/rpmesp.2016.333.2296 
pareja y el riesgo de consumo de sustancias psicoactivas en la adolescencia y la adultez ${ }^{(10,11)}$.

En la ciudad de Medellín, la segunda más desarrollada de Colombia y en la que durante diferentes periodos de la historia actual, se han presentado importantes hechos de violencia debida al narcotráfico y grupos al margen de la ley, se desarrolló un estudio con escolares de diferentes colegios públicos de la ciudad, siendo uno de sus resultados principales el hecho de contar con un $13 \%$ de menores con comportamientos altamente agresivos ${ }^{(12)}$. Otro estudio realizado en Pereira en el eje cafetero colombiano, arrojó que un $17 \%$ de los menores de primero y segundo año de primaria, matriculados en escuelas y colegios públicos presentan comportamientos agresivos (13). El presente estudio tuvo como objetivo estudiar los niveles de agresividad (entendida como la presencia de comportamientos agresivos y hostiles en el niño frente a sus pares) y prosocialidad (entendida como la capacidad del niño para hacer empatía, ayudar, compartir e invitar a otros menores a participar en actividades como el juego) en un grupo de menores escolarizados residentes en zonas urbanas y rurales de un departamento de Colombia.

\section{MATERIALES Y MÉTODOS}

\section{DISEÑO Y POBLACIÓN}

Se realizó un estudio de corte transversal, para evaluar el grado en que se presentan comportamientos agresivos (directos e indirectos) y prosociales en una muestra de 2785 niños y niñas escolarizados con edades entre los tres y los once años, residentes en trece municipios de un departamento intermedio de Colombia. Todos los menores se encontraban matriculados en escuelas de la red de educación pública, pertenecían a familias en condiciones de pobreza, la mayoría de ellas establecidas en barrios y sectores rurales con altos niveles de violencia intrafamiliar y social. La muestra fue por conveniencia debido a que los niños participantes pertenecen a instituciones cuyos maestros habían participado en intervenciones encaminadas al manejo de la agresividad infantil desde la escuela.

\section{INSTRUMENTO}

El cuestionario para la evaluación de comportamientos agresivos y prosociales (COPRAG) Anexo 1, ha sido utilizado desde el año 2000 para evaluar la presencia de comportamientos agresivos y prosociales en niños con edades entre los tres y los once años. La evaluación de la presencia de comportamientos agresivos y/o prosociales, se realiza a través de la observación de los maestros en la escuela quienes reportan información sobre la frecuencia con la que los menores realizan actos asociados a comportamientos que indican relaciones sociales conflictivas, como destruir sus propias cosas, participar en riñas, decir palabras soeces, destruir objetos, hablar mal de otras personas a espaldas de ellas, entre otros. El COPRAG mide agresividad con 18 ítems (18 a 54 puntos), de los cuales 14 miden agresividad directa (14 a 42 puntos) y 4 miden agresividad indirecta (4 a 12 puntos). El mismo instrumento permite, a su vez, evaluar la capacidad del niño para ayudar, compartir e invitar a otros menores a participar en actividades como el juego con 11 ítems (11 a 33 puntos). La escala del COPRAG establece puntos de corte solo para agresividad general y prosocialidad. Un menor es clasificado como agresivo si tiene un puntaje mayor o igual a 22 puntos, es decir, se requiere un intervalo de cuatro puntos por encima del valor mínimo (el $11,1 \%$ de los 36 puntos que componen la amplitud de la escala completa) para ser clasificado como agresivo. En este estudio proponemos puntos de corte para la agresividad directa e indirecta, haciendo una relación lineal entre los rangos de valores posibles dentro de cada subescala, con los rangos de la escala de agresividad general. Para establecer los puntos de corte de las subescalas de agresividad directa e indirecta, se tomó el rango completo de puntajes definido por la subescala y se multiplicó por 0,111 , de modo que un menor con cinco puntos o más en agresividad indirecta se clasificó como un niño que expresa solo agresividad indirecta, y un menor con 17 puntos o más en la otra subescala quedó clasificado como un niño que expresa agresividad directa. Respecto a la prosocialidad, los menores fueron clasificados como poco prosociales (por debajo de 18 puntos en la escala) y con moderada o alta prosocialidad.

Se consideró como criterio de inclusión: estar matriculado en una escuela pública cuyo director y grupo de profesores aceptaron participar en el proyecto y tener la aceptación del padre de familia, acudiente o cuidador del niño.

\section{ANÁLISIS DE DATOS}

El conjunto de datos fue dividido en cuatro grupos. Un grupo formado por menores con edades entre los tres y los cinco años (grupo 1), un segundo grupo con edades entre los seis y siete años (grupo 2), el tercero con menores cuya edad estaba entre los ocho y los nueve años (grupo 3) y el último grupo con los niños de 10 y 11 años (grupo 4). Adicionalmente, se conformaron ocho grupos considerando los cuatro grupos etarios y el sexo. De acuerdo con las puntuaciones en la escala COPRAG, se clasificó a los menores como agresivos y no agresivos para cada uno de los tipos de agresividad. Con las variables categorizadas se identificaron los menores que presentan, al menos, uno de los tipos de agresividad, y aquellos que presentan rasgos de agresividad y de prosocialidad simultáneamente. Se obtuvieron las proporciones de niños que presentaban 
las características de interés y se utilizaron pruebas chi cuadrado asumiendo un error tipo I máximo tolerable de 0,05, para establecer diferencias entre los grupos de estudios. El análisis de datos se realizó con la ayuda del SPSS versión 21 para Windows.

\section{CONSIDERACIONES ÉTICAS}

El protocolo de investigación desarrollado contó con el aval del Comité de Ética de la Universidad Tecnológica de Pereira. Los docentes convocaron a los padres de familia y asistentes de los menores, a una reunión en la que se les explicó el alcance del proyecto y los pormenores sobre la participación de sus hijos en el mismo. Los padres de familia firmaron el consentimiento informado y recibieron capacitación sobre intervenciones efectivas en la reducción del comportamiento agresivo en niños. Con los docentes se realizaron talleres acerca del manejo del comportamiento agresivo en los niños y la forma como debían diligenciar el instrumento de evaluación.

\section{RESULTADOS}

El instrumento fue aplicado a un grupo de 2785 menores de ambos sexos, 1453 niños (52\%) y 1332 niñas. Entre los menores de sexo masculino la edad media fue de 8,7 años ( $\pm 2,1$ años) y en el grupo de niñas fue de 8,8 años $( \pm 1,97$ años). El $9,2 \%$ eran menores con edades entre los tres y cinco años; el 13,2\% tenían seis o siete años de edad; el $32,2 \%$ eran menores con ocho o nueve años y el resto $(45,3 \%)$ tenían entre diez y once años. Los análisis psicométricos confirmaron las hipótesis consideradas al construir el instrumento y el valor observado del índice de Crombach $(0,90)$ coincidió con los valores obtenidos para el mismo indicador en estudios previos, realizados con diferentes muestras de menores.

Agresividad y prosocialidad: después de clasificar a los menores de acuerdo con las puntuaciones obtenidas y los puntos de corte definidos, se encontró que el 55,5\% fue clasificado dentro de la categoría de no agresivo (62,2\% de las niñas y $49,4 \%$ de los niños, $p=0,001$ ); el $18,0 \%$ del grupo presentó características asociadas solo con la agresividad directa (el $13,2 \%$ de las niñas y $22,4 \%$ de los niños, $p=0,001)$; el $5,7 \%$ presentó comportamientos propios de agresividad indirecta $(8,0 \%$ de las niñas y $3,6 \%$ de los niños, $p=0,294$ ) y el restante $20,8 \%$ presentó comportamientos propios de los dos tipos de agresividad $(16,6 \%$ de las niñas y $24,6 \%$ de los niños, $p=0,001)$.

La mayor proporción de menores con agresividad indirecta se presentó en el grupo de 8 a 9 años. Los comportamientos que expresan agresividad directa se observaron en mayor proporción en el grupo de 10 a 11 años. En el grupo de 6 a 8 años fue mayor la proporción de menores con ambas formas de agresividad. Los comportamientos prosociales fueron más comunes en las niñas y en el grupo de 10 a11 años (Tabla 1).

Al realizar el análisis entre los grupos formados por el sexo y la edad, se observó que las mayores proporciones de menores clasificados como no agresivos se presentaron en las niñas mayores de 8 años $(65,5$ y $62,3 \%$ ), mientras que entre los varones de las mismas edades, se observaron las mayores proporciones de individuos con agresividad directa (21,1 y 26,5\%), en ambos casos se obtuvo un valor $p$ de 0,001. La mayor proporción de menores con agresividad indirecta se observó entre las niñas con cinco o menos años de edad, y las mayores proporciones de comportamientos de ambas agresividades se observaron entre los varones del grupo de 6 a 7 años. Los comportamientos prosociales son más comunes entre las niñas, observándose la mayor proporción en el grupo de 10 a 11 años (Tabla 2).

Al estudiar la relación entre agresividad y prosocialidad, se pudo establecer que entre las niñas es mayor la presencia de comportamientos de agresividad indirecta y niveles

Tabla 1. Distribución de los menores de acuerdo con la edad, el sexo y la clasificación de agresividad y prosocialidad

\begin{tabular}{|c|c|c|c|c|c|c|}
\hline & \multirow[b]{2}{*}{ No agresivo } & \multirow[b]{2}{*}{$\begin{array}{l}\text { Agresividad } \\
\text { directa }\end{array}$} & \multirow[b]{2}{*}{$\begin{array}{c}\text { Agresividad } \\
\text { indirecta }\end{array}$} & \multirow[b]{2}{*}{$\begin{array}{c}\text { Ambas } \\
\text { agresividades }\end{array}$} & \multicolumn{2}{|c|}{ Prosocialidad } \\
\hline & & & & & Baja & $\begin{array}{c}\text { Moderada } \\
\text { alta }\end{array}$ \\
\hline \multicolumn{7}{|l|}{ Sexo (\%) } \\
\hline Hombre, $n=1453$ & 49,4 & 22,4 & 3,6 & 24,6 & 42,1 & 57,9 \\
\hline Mujer, $n=1332$ & 62,2 & 13,2 & 8,0 & 16,6 & 32,8 & 67,2 \\
\hline \multicolumn{7}{|l|}{ Edad en años (\%) } \\
\hline 3 a $5, n=257$ & 54,1 & 16,0 & 6,2 & 23,7 & 46,7 & 53,3 \\
\hline 6 a $8, n=368$ & 54,1 & 11,7 & 5,2 & 29,1 & 42,7 & 57,3 \\
\hline 8 a $9, n=898$ & 58,7 & 16,0 & 6,5 & 18,8 & 40,1 & 59,9 \\
\hline 10 a $11, n=1262$ & 54,0 & 21,7 & 5,2 & 19,2 & 32,6 & 67,4 \\
\hline
\end{tabular}


Tabla 2. Distribución de la expresión de agresividad y prosocialidad entre los grupos formados al combinar el sexo y la edad

\begin{tabular}{|c|c|c|c|c|c|c|}
\hline & \multirow[b]{2}{*}{ No agresivo } & \multirow[b]{2}{*}{$\begin{array}{l}\text { Agresividad } \\
\text { directa }\end{array}$} & \multirow[b]{2}{*}{$\begin{array}{l}\text { Agresividad } \\
\text { indirecta }\end{array}$} & \multirow[b]{2}{*}{$\begin{array}{c}\text { Ambas } \\
\text { agresividades }\end{array}$} & \multicolumn{2}{|c|}{ Prosocialidad } \\
\hline & & & & & Baja & $\begin{array}{c}\text { Moderada o } \\
\text { alta }\end{array}$ \\
\hline \multicolumn{7}{|l|}{3 a 5 años (\%) } \\
\hline Hombre, $n=143$ & 54,5 & 18,9 & 7,0 & 25,9 & 51,0 & 49,0 \\
\hline Mujer, $n=114$ & 53,5 & 12,2 & 13,2 & 21,1 & 41,2 & 58,8 \\
\hline \multicolumn{7}{|l|}{6 a 7 años (\%) } \\
\hline Hombre, $n=190$ & 48,9 & 14,2 & 3,7 & 33,2 & 51,1 & 48,9 \\
\hline Mujer, $n=178$ & 59,6 & 9,0 & 6,7 & 24,7 & 33,7 & 66,3 \\
\hline \multicolumn{7}{|l|}{8 a 9 años (\%) } \\
\hline Hombre, $n=487$ & 53,0 & 21,1 & 3,9 & 22,0 & 43,5 & 56,5 \\
\hline Mujer, $n=411$ & 65,5 & 10,0 & 9,5 & 15,1 & 36,0 & 64,0 \\
\hline \multicolumn{7}{|l|}{10 a 11 años (\%) } \\
\hline Hombre, $n=633$ & 45,7 & 26,5 & 3,9 & 23,9 & 36,2 & 63,8 \\
\hline Mujer, n=629 & 62,3 & 16,9 & 6,4 & 14,5 & 28,9 & 71,1 \\
\hline
\end{tabular}

altos o moderados de prosocialidad en todos los grupos de edad, mientras que entre los varones, la tendencia es a presentar comportamientos propios de agresividad directa acompañados de niveles altos o moderados de prosocialidad a medida que aumenta la edad (Tabla 3). En los casos donde fue posible realizar una prueba estadística para evaluar asociación, se observaron valores $p$ que no permitían rechazar la hipótesis de independencia entre agresividad y prosocialidad.

\section{ANÁLISIS POR ÍTEMS DE AGRESIVIDAD}

Se realizó un análisis para establecer con respecto a la frecuencia de aparición de los actos agresivos en el niño, reportada por el maestro, cuáles eran los más frecuentes

Tabla 3. Distribución de los niveles de prosocialidad y agresividad entre los grupos formados según edad y sexo

\begin{tabular}{|c|c|c|c|c|}
\hline Prosocialidad según edad y sexo & No agresivo & $\begin{array}{c}\text { Agresividad } \\
\text { directa }\end{array}$ & $\begin{array}{l}\text { Agresividad } \\
\text { indirecta }\end{array}$ & $\begin{array}{c}\text { Ambas } \\
\text { agresividades }\end{array}$ \\
\hline \multicolumn{5}{|l|}{ Niñas de 3 a 5 años, $n=114$} \\
\hline Baja (\%) & 27,2 & 6,1 & 0,9 & 7,0 \\
\hline Moderada o alta (\%) & 26,3 & 6,1 & 12,3 & 14,0 \\
\hline \multicolumn{5}{|l|}{ Niñas de 6 a 7 años, $n=178$} \\
\hline Baja (\%) & 24,2 & 2,8 & 0,0 & 6,7 \\
\hline Moderada o alta (\%) & 35,4 & 6,2 & 6,7 & 18,0 \\
\hline \multicolumn{5}{|l|}{ Niñas de 8 a 9 años, $n=411$} \\
\hline Baja & 23,4 & 3,6 & 1,5 & 7,5 \\
\hline Moderada o alta & 42,1 & 6,3 & 8,0 & 7,5 \\
\hline \multicolumn{5}{|l|}{ Niñas de 10 a 11 años $n=629$} \\
\hline Baja (\%) & 18,1 & 4,8 & 1,9 & 4,1 \\
\hline Moderada o alta (\%) & 44,2 & 12,1 & 4,5 & 10,3 \\
\hline \multicolumn{5}{|l|}{ Niños de 3 a 5 años, $n=114$} \\
\hline Baja (\%) & 31,5 & 9,1 & 0 & 10,5 \\
\hline Moderada o alta (\%) & 23,1 & 9,8 & 0,7 & 15,4 \\
\hline \multicolumn{5}{|l|}{ Niños de 6 a 7 años, $n=178$} \\
\hline Baja (\%) & 27,9 & 6,8 & 1,6 & 14,7 \\
\hline Moderada o alta (\%) & 21,1 & 7,4 & 2,1 & 18,4 \\
\hline \multicolumn{5}{|l|}{ Niños de 8 a 9 años, $n=411$} \\
\hline Baja (\%) & 26,5 & 8,0 & 1,6 & 7,4 \\
\hline Moderada o alta (\%) & 26,5 & 13,1 & 2,3 & 14,6 \\
\hline \multicolumn{5}{|l|}{ Niños de 10 a 11 años, $n=629$} \\
\hline Baja (\%) & 16,1 & 8,8 & 1,1 & 10,1 \\
\hline Moderada o alta (\%) & 29,5 & 17,7 & 2,8 & 13,7 \\
\hline
\end{tabular}


Tabla 4. Distribución de los menores para la respuesta "siempre" a los ítems de agresividad indirecta

\begin{tabular}{|c|c|c|c|c|c|c|c|}
\hline & $\begin{array}{l}\text { Grupo } \\
\text { Completo }\end{array}$ & Niñas & Niños & $\begin{array}{c}\text { Edad } \\
3-5\end{array}$ & $\begin{array}{c}\text { Edad } \\
6-7\end{array}$ & $\begin{array}{c}\text { Edad } \\
8-9\end{array}$ & $\begin{array}{l}\text { Edad } \\
10-11\end{array}$ \\
\hline $\begin{array}{l}\text { Ítem 7. Cuando esta bravo con alguien intenta que } \\
\text { otros hagan lo mismo con esa persona (\%) }\end{array}$ & 8,5 & 7,8 & 9,2 & 9,7 & 7,9 & 8,4 & 8,6 \\
\hline $\begin{array}{l}\text { Ítem 14. Cuando esta bravo con alguien se hace } \\
\text { amigo de otros como venganza }(\%)\end{array}$ & 8,2 & 7,7 & 8,7 & 8,6 & 7,1 & 6,8 & 9,5 \\
\hline $\begin{array}{l}\text { Ítem 21. Cuando esta disgustado con alguien dice } \\
\text { cosas en su contra a sus espaldas (\%) }\end{array}$ & 10,6 & 11,0 & 10,3 & 5,4 & 9,0 & 4,6 & 12,9 \\
\hline $\begin{array}{l}\text { Ítem 31. Cuando esta disgustado con alguien le dice } \\
\text { a los demás que no compartan con esa persona (\%) }\end{array}$ & 7,5 & 7,3 & 7,7 & 7,0 & 10,1 & 6,9 & 7,3 \\
\hline
\end{tabular}

en los diferentes grupos formados para el estudio. Se consideró aquellos comportamientos que los maestros reportaron ser expresados "SIEMPRE" por el niño, como aquellos indicadores de agresividad (Tabla 4 y 5). El comportamiento asociado con agresividad indirecta con mayor frecuencia de aparición entre los menores fue el descrito en el ítem 21 del instrumento "Cuando está disgustado con alguien dice cosas en su contra a sus espaldas" cuya frecuencia más alta fue observado en el grupo de niñas (Tabla 4). Respecto a la agresividad directa, el comportamiento más frecuentemente observado fue el descrito en el ítem 20 "Cuando otro niño accidentalmente golpea asume que quiso hacerle daño y reacciona con rabia y pelea" con una proporción importante entre los varones y porcentajes similares en todos los grupos de edad. Otros ítems con comportamientos cuya frecuencia puede considerarse importante entre los niños varones son el 22, 25 y 40 , este último con proporciones importantes entre los niños de mayor edad. El ítem 35 presenta una frecuencia alta entre los menores con edad entre los tres y los cinco años Tabla 5.

Tabla 5. Distribución de los menores para la respuesta "SIEMPRE" a los ítems de agresividad directa

\begin{tabular}{|c|c|c|c|c|c|c|c|}
\hline & $\begin{array}{l}\text { Grupo } \\
\text { Completo }\end{array}$ & Niñas & Niños & Edad 3-5 & Edad 6-7 & Edad 8-9 & Edad 10-11 \\
\hline Ítem 1. Destruye sus propias cosas (\%) & 5,4 & 4,6 & 6,1 & 8,6 & 5,7 & 5,0 & 4,9 \\
\hline Ítem 2. Se mete en muchas peleas (\%) & 10,9 & 6,4 & 15 & 15,2 & 10,9 & 9,8 & 10,8 \\
\hline $\begin{array}{l}\text { Ítem } 9 \text {. Destruye objetos que pertenecen a } \\
\text { otros niños }(\%)\end{array}$ & 5,6 & 3,4 & 7,6 & 7,8 & 7,3 & 4,7 & 5,3 \\
\hline Ítem 16. Dice mentiras, hace trampa (\%) & 9,2 & 7,4 & 10,8 & 5,4 & 7,6 & 10 & 9,8 \\
\hline $\begin{array}{l}\text { Ítem 20. Cuando otro niño accidentalmente } \\
\text { golpea asume que quiso hacerle daño y } \\
\text { reacciona con rabia y pelea (\%) }\end{array}$ & 14,6 & 10,4 & 18,6 & 14,0 & 16,0 & 15,5 & 13,8 \\
\hline Ítem 22. Ataca físicamente a las personas (\%) & 8,4 & 5,9 & 10,7 & 8,2 & 10,9 & 7,3 & 8,6 \\
\hline $\begin{array}{l}\text { Ítem } 25 . \text { Amenaza con gestos o palabras a } \\
\text { otros (\%) }\end{array}$ & 8,9 & 6,8 & 10,9 & 8,9 & 7,3 & 9,6 & 9,0 \\
\hline $\begin{array}{l}\text { Ítem 29. Es cruel, abusivo o malvado con otras } \\
\text { personas o con animales (\%) }\end{array}$ & 4,7 & 3,0 & 6,2 & 3,5 & 5,7 & 4,0 & 5,1 \\
\hline $\begin{array}{l}\text { Ítem 33. Patea, muerde, golpea a otros niños } \\
(\%)\end{array}$ & 6,0 & 3,9 & 7,8 & 8,2 & 5,7 & 6,0 & 5,5 \\
\hline $\begin{array}{l}\text { Ítem 35. Toma objetos de otros niños sin su } \\
\text { permiso (\%) }\end{array}$ & 50 & 3,2 & 6,5 & 10,9 & 6,3 & 4,5 & 3,7 \\
\hline Ítem 38. Imita el llanto de otros niños (\%) & 5,5 & 3,8 & 7,1 & 3,1 & 4,3 & 5,7 & 6,7 \\
\hline $\begin{array}{l}\text { Ítem } 40 \text {. Se ríe de los errores de otros niños } \\
(\%)\end{array}$ & 9,0 & 6,6 & 11,2 & 2,7 & 7,1 & 9,2 & 10,7 \\
\hline $\begin{array}{l}\text { Ítem 42. Se ríe de las limitaciones o defectos } \\
\text { de otros niños (\%) }\end{array}$ & 6,6 & 4,3 & 8,7 & 0,8 & 6,0 & 7,0 & 7,6 \\
\hline $\begin{array}{l}\text { Ítem 44. Daña juegos o actividades de los } \\
\text { otros (\%) }\end{array}$ & 6,3 & 4,9 & 7,6 & 6,6 & 8,4 & 7,3 & 4,8 \\
\hline
\end{tabular}




\section{DISCUSIÓN}

La presencia de comportamientos agresivos altera el desempeño académico de niños y niñas ya que, por presentarlos, los niños pueden ser excluidos de los procesos educativos en sus escuelas ${ }^{(14,15)}$. La exclusión del aula está asociada a que se manifiesten de manera más clara y frecuente actitudes hostiles y violentas frente a las demás personas en el entorno social y familiar del menor. Esta ruta define, en el tiempo, que los niños se conviertan en adultos de bajo desempeño académico y determinando que por ello tengan una condición social de igual o mayor pobreza que la de sus padres, lo que se define como transmisión intergeneracional de pobreza ${ }^{(16,17)}$.

Los niños en edad escolar al considerar que las reglas impuestas por los padres de familia y docentes son normas que deben ser cumplidas, de modo que, cuando hay una relación interpersonal adecuada, los adultos son considerados por parte de los niños, como figuras significativas, es decir, personas con fuerte vínculo afectivo con los niños a los cuales se les cree lo que se verbaliza. De acuerdo con Bandura 1986, entre los siete a los once años, los niños adquieren la capacidad de realizar desarrollos mentales con los objetos que tienen delante, sin poder hacer generalizaciones abstractas, son conscientes de que algunas normas establecen relaciones entre las cosas, entonces, el niño si tiene un buen desarrollo podrá llegar a concluir que las normas pueden ser la base para el desarrollo del respeto mutuo entre sus compañeros de juego. Una forma de monitorear el desarrollo del menor, es poder definir el nivel de presencia de comportamientos prosociales y agresivos. Los comportamiento agresivos directos se expresan a través de la motricidad, son comportamientos motores ${ }^{(13)}$ asociados a actos que en algunos contextos pueden considerarse de violencia física. Los niños con este tipo de comportamientos no son aceptados por los pares y fácilmente son definidos como agresivos por los adultos ${ }^{(18)}$. Respecto a los comportamientos agresivos indirectos, estos parecen ser más propios de las niñas que de los niños, resultado observado en este estudio y que coinciden con los obtenidos en otras investigaciones ${ }^{(13,19)}$. En este caso, el menor expresa su comportamiento agresivo a través de terceros y en forma más verbal ${ }^{20)}$. Los niños y niñas con mayor presencia de agresividad indirecta, serán más difíciles de identificar por los adultos ya que pueden pasar desapercibidos, a no ser que los adultos se tomen el trabajo de observarlos en los juegos y trabajos en grupo. Una expresión de ese comportamiento agresivo indirecto se presenta cuando participan de la reducción del prestigio de pares o de adultos sin que ellos estén presentes para defenderse ${ }^{(21,22)}$.

El comportamiento agresivo puede ser modificado con intervenciones comunitarias buscando una reducción de la presencia de conductas y comportamientos agresivos en el infante, al mismo tiempo que se estimula la aparición y expresión de comportamientos prosociales que le permitirían a los niños mejores relaciones con sus pares y los adultos ${ }^{(23,24,25)}$. Aun cuando, la frecuencia de comorbilidad con algunos trastornos como el déficit de atención es muy baja, se considera importante identificar a los niños con comportamientos muy agresivos y remitirlos a valoración por diferentes profesionales de la salud (médico, psicólogo, terapeuta ocupacional etc.) con el fin de hacer un análisis que permita, a través de sus antecedentes y entornos familiares, identificar posibles causas o factores que expliquen su comportamiento al tiempo que sea posible establecer su nivel de desarrollo y qué tan adecuado es el mismo, además de implementar alternativas terapéuticas adecuadas. Algunos autores como Klevens et al. y Love et al. coinciden en afirmar que la escuela puede ser vista como un espacio especialmente privilegiado para intervenir positivamente a los menores con comportamientos agresivos. Se pueden asumir estrategias transcurriculares apoyadas en modelos que estimulen la dinámica del desarrollo cognitivo individual, puesto que el niño, al compartir su cotidianidad con individuos semejantes, se torna particularmente susceptible de aprender y asimilar conductas e ideas de ellos (una de las vías primordiales de desarrollo de los niños es la identificación con sus pares). Desde esta perspectiva, la escuela se convierte en escenario privilegiado para la transmisión de patrones violentos o pacíficos de interrelación ${ }^{(26)}$. Desde muy temprana edad el niño aprende y rápidamente, pero, particularmente, el desarrollo de habilidades emocionales ${ }^{(27)}$. Por ejemplo, se ha establecido que el vínculo afectivo se tiene desde antes del año y la funcionalidad de ese vínculo es un factor protector que estimula unas adecuadas relaciones interpersonales ${ }^{(28)}$.

Al igual que muchas zonas de Colombia, la región de la que se tomó la muestra de estudio, se ha visto expuesta a diferentes expresiones de violencia (desplazamiento forzado, combates, violencia intrafamiliar etc.) que aunadas a las condiciones de pobreza, conllevan a que muchos de los las habitan presenten altos niveles de agresividad, que son reflejados en forma de comportamientos y actitudes hostiles frente a los semejantes y, en algunas ocasiones, frente a sí mismos. De acuerdo con los resultados obtenidos, es posible concluir que los niños tienden a presentar más comportamientos agresivos motores (agresividad directa) y las niñas más comportamientos agresivos verbales (agresividad indirecta). Una explicación plausible podría enfocarse desde el punto de vista de las condiciones de entorno, contexto familiar y sociocultural del menor.

Todos los niños participantes en esta investigación pertenecen a familias que habitan en condiciones de pobreza extrema zonas periféricas de la ciudad o en zonas rurales del departamento, las cuales guardan en común el hecho de haber estado expuestas en distintos grados a expresiones violentas debidas al conflicto de convivencia que se vive y que durante varias décadas ha estado presente en el país. Ese factor, unido al hecho de tener condiciones culturales que permiten aceptar normas de convivencia como que las niñas no deben golpear ni gritar a sus semejantes, porque ellas son "femeninas", mientras que los niños pueden usar los medios de agresión física porque son "hombres", pueden, entre muchas otras explicaciones posibles, dar razones para entender cómo las expresiones de 
agresividad pueden ser una cuestión de género; sin embargo, consideramos que podrían realizarse estudios más profundos acerca del tema explorando otros posibles factores que estén relacionados con la presencia de los diferentes tipos de agresividad en niños y niñas. Este tipo de modelos no permite que se estimule la memoria del buen comportamiento pasado impidiendo el refuerzo de comportamientos prosociales que son infrecuentes y poco estimulados en estas comunidades ${ }^{(29)}$. Las relaciones estimuladas a través de los medios, y en particular de la televisión, son relaciones basadas más en conflictos que la en resolución de limita a los niños en sus opciones de modelos de tipos relaciones de las personas basadas en comportamientos prosociales ${ }^{(30)}$.
Contribuciones de autoría: JWM escribió la introducción y discusión, revisión de la literatura, recolección de datos en campo y lectura crítica del documento; JRTV escribió la sección de materiales y métodos resultados y conclusiones, AFO participo del procesamiento y análisis estadístico de los datos y obtención de resultados

\section{Fuentes de financiamiento: autofinanciado}

Conflictos de interés: los autores declaran no tener conflictos en la publicación del artículo.

Anexos disponibles en: http://www.rpmesp.ins.gob.pe/index. php/rpmesp/article/view/2296/2210

\section{REFERENCIAS BIBLIOGRÁFICAS}

1. Farrington DP. The development of offending and antisocial behaviour from childhood: Key findings from the Cambridge Study in Delinquent Development. J Child Psychol Psychiatry. 1995;36(6):929-64.

2. Farrington DP, Loeber R. Epidemiology of juvenile violence. Child Adolesc Psychiatr Clin N Am. 2000;9(4):733-48.

3. Krug EG, Dahlberg LL, Mercy JA, Zwi $A B$, Lozano R. Informe mundial sobre la violencia y la salud. Washington, D.C.: 2003. p. 374

4. Masse LC, Tremblay RE. Behavior of boys in kindergarten and the onset of substance use during adolescence. Arch Gen Psychiatry. 1997;54(1):62-8.

5. Kalb LM, Loeber R. Child disobedience and noncompliance: a review. Pediatrics.2003;111(3):641-52.

6. Tolan P, Guerra N. What works in reducing adolescent violence: an empirical review of the field. Boulder: Institute of Behavioral Science; 1994.

7. Tremblay RE, Nagin DS, Séguin JR, Zoccolillo M, Zelazo PD, Boivin M, et al. Physical aggression during early childhood: Trajectories and predictors. Pediatrics. 2004;114(1):e43-50.

8. Hutchings J, Gardner F, Bywater T, Daley D, Gardner F, Whitaker C, et al. Parenting intervention in Sure Start services for children at risk of developing conduct disorder: pragmatic randomised controlled trial. BMJ. 2007;334(7595):678.

9. Tremblay RE, McCord J, Boileau $\mathrm{H}$, Charlebois $\mathrm{P}$, Gagnon C, Le Blanc M, et al. Can disruptive boys be helped to become competent? Psychiatry. 1991;54(2):148-61.

10. Centers for Disease Control and Prevention. School health guidelines to prevent unintentional injuries and violence. MMWR Recomm Rep. 2001;50(RR-22):1-73.

11. Durlak JA, Wells AM. Primary prevention mental health programs for children and adolescents: a meta-analytic review. Am J Community Psychol. 1997 Apr;25(2):115-52.
12. Duque LF, Orduz JF, Sandoval Jde J, Caicedo BE, Klevens J. Lecciones del programa de prevención temprana de la violencia, Medellín, Colombia. Rev Panam Salud Publica. 2007;21(1):21-9.

13. Martinez JW, Rojas C, Duque A, Tovar $\mathrm{R}$, Klevens J. ¿'Son los niños más agresivos que las niñas? Comportamiento de la agresividad en niños y niñas de escuelas públicas de Pereira. Rev Med Risaralda. 2008.14(1):23-30

14. Chung I-J, Hill KG, Hawkins JD, Gilchrist LD, Nagin DS. Childhood predictors of offense trajectories. JRCD. 2002;39(1):60-90. doi: $10.1177 / 002242780203900103$.

15. Díaz J. La violencia en la escolar: diagnóstico y prevención. Rev Psiquiatr Psicol Niño Adolesc. 2001;1(1):57-79.

16. Engle PL, Black MM, Behrman JR, Cabral de Mello M, Gertler PJ, Kapiriri $\mathrm{L}$, et al. Strategies to avoid the loss of developmental potential in more than 200 million children in the developing world. Lancet. 2007;369(9557):229-42.

17. Grantham-McGregor S, Cheung YB, Cueto S, Glewwe P, Richter L, Strupp B, et al. Developmental potential in the first 5 years for children in developing countries. Lancet. 2007;369(9555):60-70.

18. Klevens J, Martínez JW, Le B, Rojas C, Duque A, Tovar R. Evaluation of two interventions to reduce aggressive and antisocial behavior in first and second graders in a resource-poor setting. Int J Educ Res. 2009;48(5):307-19. doi: 10.1016/j.ijer.2010.03.001.

19. Nagin D, Tremblay RE. Trajectories of boys physical aggression, opposition, and hyperactivity on the path to physically violent and nonviolent juvenile delinquency. Child Dev. 1999;70(5):1181-96.

20. Marcus RF, Kramer C. Reactive and proactive aggression: attachment and social competence predictors. J Genet Psychol. 2001;162(3):260-75.

21. Martínez JW. Intervención de salud pública para la prevención o control de la violencia. Investigaciones Andina. 2004; (8):36-40

22. Reese LE, Vera EM, Simon TR, Ikeda RM. The role of families and care givers as risk and protective factors in preventing youth violence. Clin Child Fam Psychol Rev. 2000;3(1):61-77.

23. Greenberg MT, Kusche CA, Cook ET, Quamma JP. Promoting emotional competence in school-aged children: The effects of the PATHS curriculum. Dev Psychopathol.1995;7(01):117-36. doi: $10.1017 /$ S0954579400006374.

24. Greenwood CR, Hops H, Walker HM, Guild JJ, Stokes J, Young KR, et al. Standardized classroom management program: Social validation and replication studies in Utah and Oregon.J Appl Behav Anal. 1979;12(2):235-53.

25. Hahn R, Fuqua-Whitley D, Wethington $\mathrm{H}$, Lowy J, Crosby A, Fullilove M, et al. Effectiveness of universal school-based programs to prevent violent and aggressive behavior: a systematic review. Am J Prev Med. 2007;33(2 Suppl):S114-29.

26. Sampson RJ, Raudenbush SW, Earls F. Neighborhoods and violent crime: A multilevel study of collective efficacy. Science. 1997; 277(5328):918-24.

27. Nie Y-G, Li J-B, Vazsonyi AT. Self-control mediates the associations between parental attachment and prosocial behavior among Chinese adolescents. Pers Individ Dif.2016;96:36-9.

28. Montgomery P, Maunders K. The effectiveness of creative bibliotherapy for internalizing, externalizing, and prosocial behaviors in children: A systematic review. Child Youth Serv Rev. 2015;55:37-47. doi:10.1016/j.childyouth.2015.05.010.

29. Tasimi A, Young L. Memories of good deeds past: The reinforcing power of prosocial behavior in children. J Exp Child Psychol. 2016;147:159-66. doi: 10.1016/j.jecp.2016.03.001.

30. Xie X, Chen W, Lei L, Xing C, Zhang Y. The relationship between personality types and prosocial behavior and aggression in Chinese adolescents. Pers Individ Dif. 2016;95:56-61. doi: 10.1016/j.paid.2016.02.002.

Correspondencia: José Rafael Tovar Cuevas

Dirección: Calle 13 No 100-00. Santiago de CaliColombia

Teléfono: 3212100 ext 114

Correo electrónico:jose.r.tovar@correounivalle.edu.co 


\section{ANEXO}

Anexo1: Evaluación del comportamiento agresivo y prosocial de niños y niñas escolarizados (Coprag)

Nombre del niño:

Nombre del cuidador o acudiente o padre de familia:

Nombre de la escuela:

Grupo:

Edad:

Sexo:

Fecha:

\begin{tabular}{|c|c|c|c|}
\hline Ítems & Nunca 1 & Algunas veces 2 & Casi siempre 3 \\
\hline Destruye sus propias cosas & & & \\
\hline Ayuda a quien se ha golpeado & & & \\
\hline Se mete en muchas peleas & & & \\
\hline 4. Se ofrece a arreglar desórdenes & & & \\
\hline $\begin{array}{l}\text { 5. Cuando esta bravo(a) con alguien, intenta que otros hagan lo mismo } \\
\text { con esa persona }\end{array}$ & & & \\
\hline $\begin{array}{l}\text { 6. Destruye (romper, derribar o pisar voluntariamente) objetos que } \\
\text { pertenecen a su familia u otros niños }\end{array}$ & & & \\
\hline 7. Si hay alegato o pelea, tratará de detenerla & & & \\
\hline $\begin{array}{l}\text { 8. Cuando esta bravo(a) con alguien se hace amigo de otros como } \\
\text { venganza }\end{array}$ & & & \\
\hline 9. $\quad$ Dice mentiras, hace trampas & & & \\
\hline $\begin{array}{l}\text { 10. Ofrece ayuda a otros niños o niñas (amigos, hermanos) cuando tienen } \\
\text { dificultades con una labor }\end{array}$ & & & \\
\hline $\begin{array}{l}\text { 11. Cuando otro niño accidentalmente le golpea, asume que este quiso } \\
\text { hacerle daño y reacciona con rabia y pelea }\end{array}$ & & & \\
\hline $\begin{array}{l}\text { 12. Cuando esta disgustado(a) con alguien dice cosas en su contra a sus } \\
\text { espaldas }\end{array}$ & & & \\
\hline 13. Ataca físicamente a las personas & & & \\
\hline 14. Consuela niños(as) que estén llorando o tristes & & & \\
\hline 15. Amenaza con gestos o palabras a otros & & & \\
\hline $\begin{array}{l}\text { 16. Espontáneamente ayuda a recoger objetos que otros niños(as) han } \\
\text { dejado caer. Ejemplo; lápices, cuadernos, etc. }\end{array}$ & & & \\
\hline 17. Es cruel, abusivo(a) o malvado(a) con otras personas o con los animales & & & \\
\hline $\begin{array}{l}\text { 18. Cuando esta disgustado(a) con alguien le dice a los demás que no } \\
\text { comparta con esa persona }\end{array}$ & & & \\
\hline 19. Patea, muerde, golpea a otros niños(as) & & & \\
\hline 20. Invita a observadores a participar en el juego & & & \\
\hline $\begin{array}{l}\text { 21. Toma objetos de otros niños sin su permiso. (apropiación, ruptura de } \\
\text { objetos o juegos cuando un niño(a) quita a otro(a) -bien de su mano o de } \\
\text { su espacio- un objeto que estaba usando y que no es ofrecido }\end{array}$ & & & \\
\hline 22. Ayuda a otros niños(as) que se sienten mal & & & \\
\hline 23. Imita el llanto de otros niños(as) & & & \\
\hline 24. Elogia el trabajo de niños(as) menos hábiles & & & \\
\hline 25. Se ríe de los errores de otros niños(as) & & & \\
\hline 26. Intenta calmar a los niños(as) cuando están agresivos(as) & & & \\
\hline 27. Se ríe de limitaciones o defectos de otros niños(as) & & & \\
\hline 28. Comparte sus pertenencias con otros niños(as) & & & \\
\hline 29. Daña juegos o actividades de los otros & & & \\
\hline
\end{tabular}

